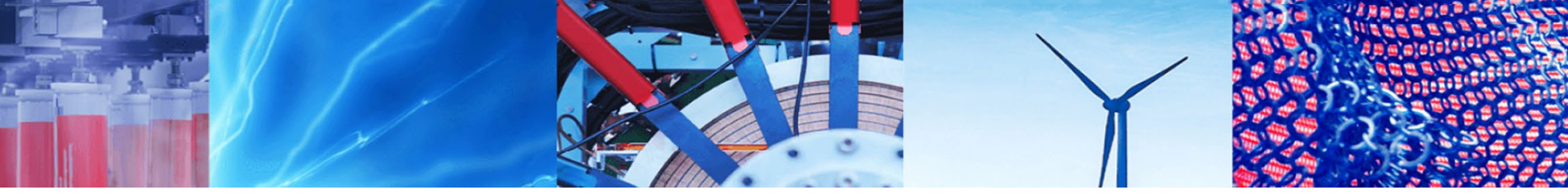

Research Article

\title{
Band pass filter plan in fluoroscopy for high energy range
}

\author{
Abdollah Khorshidi $^{1}$ (D) Ahmad Abdollahi ${ }^{1} \cdot$ Aliyar Pirouzi $^{1} \cdot$ S. Hamed Hosseini ${ }^{1,2}$
}

Received: 31 October 2019 / Accepted: 10 December 2019 / Published online: 16 December 2019

(c) Springer Nature Switzerland AG 2019

\begin{abstract}
In fluoroscopy, the absorption edge, the energy range, and the X-ray spectrum peak are the most important factors in filtration to obtain a proper image. Here, an experimental study was conducted to determine the filters that remove low-energy spectrums and attenuate high-energy ranges so that they cannot fundamentally affect image quality and diminish the absorbed dose by the patient. Considering the attenuation curves of proper elements besides accessibility and productivity issues, three elements were investigated that comprised Copper, Lead and Tin with diverse thicknesses in the X-ray energy range of 100 to $125 \mathrm{keV}$. Also, a simulation study by Monte Carlo N-Particle code was performed with an accuracy of $99 \%$. The findings demonstrated that the use of a $0.1 \mathrm{~mm}$ lead filter retains the best image quality along with a significant reduction in the dose ratio obtained by raising milliampere. Multi or auxiliary filters require additional testing to achieve better image quality. In order to obtain the best possible X-ray band pass spectrum, the analysis of the attenuation and absorption profiles of the various elements plays an essential role in the calculation of the output intensities.
\end{abstract}

Keywords Lead plate $\cdot$ Band pass filter $\cdot$ Attenuation profile $\cdot$ Quality of image $\cdot$ MCNP4C

\section{Introduction}

The relative composition of an X-ray spectrum in terms of bremsstrahlung and radiation properties depends on anode material, filtration and $\mathrm{kV}$ which comprises a diverse region of wavelengths used in fluoroscopy. While the bandwidth is lower, a better image can be obtained with respect to resolution, contrast, noise and beam hardening artifacts [1-3]. Recently, numerous studies have led to the emergence of new technologies in the field of medical imaging, and have also incurred a huge cost considering expensive new equipment, which is due to the fact that the improvement of image quality in the diagnosis plays a vital role in diseases treatment via dose reduction [4-7]. Thus, in order to obtain the best possible X-ray band pass spectrum, the analysis of attenuation and absorption profiles of different elements has an essential position in computing the output intensities using selection of the finest as a filter. At present, $\mathrm{X}$-ray low-band filters are widely used in medical centers [8-11]. With these filters, the passage of high-energy photons could be prevented without affecting or impairing the image quality [12-16].

Leschka et al. [17] found that applying tin filters in dualenergy $\mathrm{CT}$ leads to improved sensitivity and specificity for the differentiation of renal cysts from the improvement of the solid mass in a kidney phantom model. Also, Suntharalingam et al. [18] have shown in their phantom study that unimproved thoracic CT executed at $100 \mathrm{kVp}$ or $150 \mathrm{kVp}$ with tin filtration allows a reduction in the adult radiation dose, but not in children and neonates. Then, they have proposed a tube voltage between 70 and $110 \mathrm{kVp}$ for the tested radiation dose. Here, a tube potential between 100 and $120 \mathrm{kVp}$ is recommended for preschool children. Meanwhile, May et al. [19] have shown that spectral shaping by pre-filtration of tin plates allows for an average dose reduction of $17 \%$ without spoiling

$\triangle$ Abdollah Khorshidi, abkhorshidi@yahoo.com |' ${ }^{1}$ School of Paramedical, Gerash University of Medical Sciences, P.O. Box: 7441758666, Gerash, Iran. ${ }^{2}$ Department of Radiology and Radiotherapy, School of Allied, Tehran University of Medical Sciences, Tehran, Iran. 
diagnostic image quality in low-dose parasinus $\mathrm{CT}$ with $100 \mathrm{kVp}$ tube voltages. Moreover, Kim et al. [20] have demonstrated that the combination of tungsten, barium, silicon and molybdenum had a $0.3 \mathrm{~mm}$ lead tantamount dose and that the particle porosity and packing were $12-22 \%$ and $28-36 \%$, respectively. Nonetheless, a satisfactory shield-ability could be achieved with a particle packing of $30 \%$ and a porosity $>20 \%$. Consequently, it is a potential replacement for lead sheet and can be taken into account as a suitable medical radiation protection sheet with a fine economic feasibility. This can be achieved with a combination of milliampere $(\mathrm{mA}) \mathrm{s}, \mathrm{kVp}$, collimation and additional copper filtration. Some recent studies [21-23] have shown that image quality remains the same or even better when adding a copper filter accompanied by lower dose. This is one reason to examine image quality and dose in pediatric pelvic tests. To prove that the image quality remains acceptable, it is important to perform a visual and physical assessment [24].

Here, the fundamental problem is that each filtration increases the load on the X-ray tube, and thus decreases its working life. It can be scrutinized impressiveness of band pass filters in enhancing image the quality and reducing the absorbed patient dose and staff, gives the filter is correctly chosen. Hence, this research considers how to compute parameters and how to distinguish the best filter for $100 \mathrm{keV}$, which is the conventional voltage in general diagnostic radiology $[25,26]$.

\section{Methods}

According to Fig. 1a, the X-ray absorption curves were used to settle the correct filter. When we want to employ $\mathrm{X}$-ray band pass filters, low-energy and high-energy spectra are eliminated or attenuated so that a certain work area remains in the spectrum peak, which is the preferred area for medical imaging. In addition, reducing the absorbed dose is another important result of the removal of low-energy spectra. Based on the lead attenuation profiles in Fig. $1 \mathrm{~b}$ and the investigation of the energies of the elements at the absorption edge ( $\mathrm{k}$ ) and considering the X-ray spectrum peak, we can basically designate the appropriate elements and then the relevant filters. Therefore, by executing computations, the output X-ray spectra can be specified after the correct filters are applied, and then the improvement of the image specifications and the reduction of the output dose can be examined. The challenging problem of the stage is the selection of the correct filter thickness in terms of availability factors and cost effectiveness. It should be indicated that the absorption of $X$-ray raises with the third power of the atomic number. Thus, if the
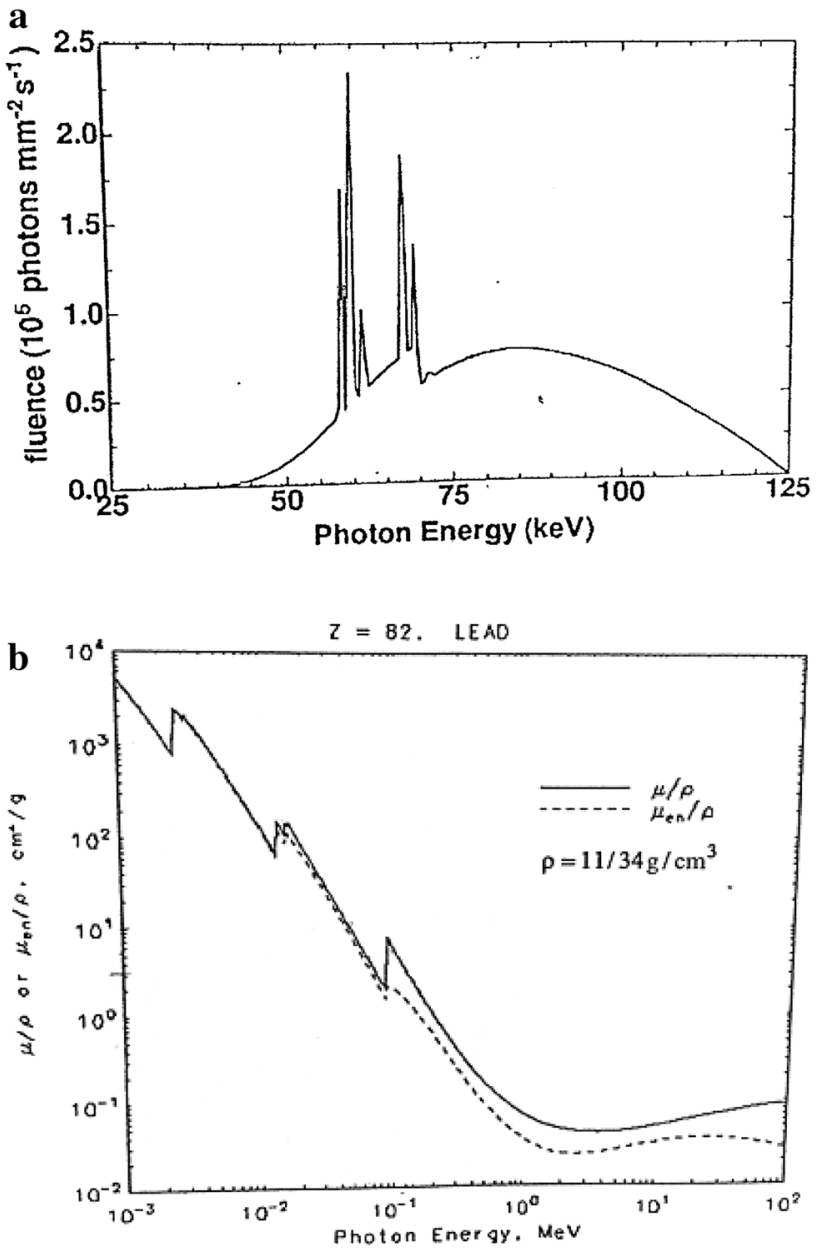

Fig. 1 a The X-ray source derived from tungsten rotating anode by focal point of $0.6 \mathrm{~mm}, 120 \mathrm{kVp}, 28 \mathrm{mAs}$, and a $1.7 \mathrm{~mm}$ thick copper filter [25] (with permission from the author to publish the photo). $\mathbf{b}$ The attenuation profile for lead filter [26] (with permission from the author to publish the photo)

absorption edge of the desired element corresponds accurately to the peak energy of our spectrum and field of action, this leads to a strong attenuation of the highenergy photons and a thinning out of the $\mathrm{X}$-ray spectrum besides a reduction of the extra absorbed patient dose through lessening low-energy photons.

We utilized the $X$-ray tube with rotating tungsten anode and a focal point of $0.6 \mathrm{~mm}, 18 \mathrm{~mA}$ current, $125 \mathrm{kVp}$ voltage, and a $1.7 \mathrm{~mm}$ thick copper filter. In this situation we want to take away photons with energy of more than $90 \mathrm{keV}$. Base on the attenuation profiles of the various elements, the $K$ absorption edge of lead $(Z=82)$ is $88 \mathrm{keV}$. Therefore, choosing lead as a filter in the desired energy range is a positive choice $[27,28]$. The attenuation coefficient of lead has been shown in Fig. $1 \mathrm{~b}$ at the range of $10^{-3}-10^{2} \mathrm{MeV}$. The thickness, $x$, needed for the element is given using the following equation regarding the intensity 
attenuation in the spectrum peak from the Beer-Lambert law (Fig. 1a):

$I=I_{0} e^{-\mu x}$

In this case, $I_{0}$ is the initial intensity of the $X$-ray beam, $I$ is the intensity of the $X$-ray beam passing through the thickness $x$ of the materials examined, and $\mu$ is the linear absorption coefficient of the material under investigation.

The Monte Carlo N Particle version 4C (MCNP4C) was used for the simulation. MCNP4C is a universal Monte Carlo radiation transport code with three dimensional geometry and continuous energy transport of ions and particles. It includes flexible source and output options (tally), interactive graphics (Vised software), and upholds both sequential and multi-processing computer stages. Simulations were performed to obtain the output spectra before and after the assignment of the filters in front of the X-ray tube with different thicknesses by examining the results within the aforesaid energies. The code was run for ten defined volumes with distinct allocated material, and then the density $\left(\mathrm{g} / \mathrm{cm}^{3}\right)$ of each region was calculated. After that, a sphere with a radius of $40 \mathrm{~cm}$ was defined at the origin, and the body of the simulation was typified by a rectangular parallelepiped (rpp) of 200 to $900 \mathrm{~cm}$, which is related to the definition of the cube-shaped from the coordinate axis. These cubes were positioned at a distance of $0.1 \mathrm{~mm}$ and represented the thickness of the filters utilized in each area. In addition, a $0.1 \mathrm{~mm}$ thick lead filter and a thin $0.1 \mathrm{~mm}$ thick tungsten target were chosen for the location where the photons pass and collide to be attenuated $[29,30]$. The input electron energy was assigned to $100 \mathrm{keV}$ and the direction of the particles incident on the target was in the vertical direction to the $Z$ axis. The simulation was performed for 10 million particles ranging from 5 to $100 \mathrm{keV}$ at an interval of 30 .

The surgical C-arm (APELEM-DMS GROUP, APX HF III series, Parc Scientifique Georges Besse, France) was used in experimental method. A C-arm intensifies the image as a scanner. This name derives from the C-shaped arm, which connects the $\mathrm{X}$-ray detector and the $\mathrm{X}$-ray source. The $\mathrm{C}$-arms have radiological capabilities, although they are mainly employed for fluoroscopic intra-operative imaging in surgical, emergency care, and orthopedic protocols. This device gives real-time and high-resolution $X$-ray images, allowing the clinician to pursue progress and make corrections immediately. The energy applied to the fluoroscopic part was $100 \mathrm{kVp}$ beside a small focus of 0.1 to $3 \mathrm{~mA}$, a constant voltage, and the machine current was raised following each exposure. In a radiographic style, an alpha phantom was located on the holder to measure the contrast, resolution, and quality. Following each exposure, the resolution and quality of the obtained images were examined in automatic mode, and the output dose was read in the third reading sector from the piranha detector, which was located in the palm rest. Then, utilizing a lead sheet thickness of $0.2 \mathrm{~mm}$ at a size of $10 \mathrm{~cm} \times 10 \mathrm{~cm}$ at the beam exit, the dose ratio, quality of the image, resolution, and contrast were examined. Figure 2 shows the device and setting situations.

\subsection{Piranha detector characteristics and calibration}

The internal Piranha detector is very sensitive and can measure the peak tube voltage for as low outputs as $50 \mathrm{kV} / 0.050 \mathrm{~mA}$ at a distance of $50 \mathrm{~cm}$. By calibration in estimation of total filtration, $1-9 \mathrm{~mm} \mathrm{Al}$ and $60-120 \mathrm{kV}$ range was used and the inaccuracy was $\pm 10 \%$ or $\pm 0.3 \mathrm{~mm}$ as well as $\pm 15 \%$ by $50 \mathrm{~mm} \mathrm{Al}$. Here, the obtained resolution was 0.1 or $1 \mathrm{~mm}$ by more than $10 \mu \mathrm{Gy} / \mathrm{s}$ dose ratio.

After positioning the Piranha detector in front of the image intensifier, the Position Check should be used to confirm the position of the detector. When fluoroscopy is at play, the Piranha system automatically updates the display using the highest possible sensitivity.

On the other hand, the external Dose Probe is normally used to measure the lowest possible dose rate levels down to $0.1 \mu \mathrm{Gy} / \mathrm{s}$. Another reason for using the external Dose Probe is that the detector is much smaller than the Piranha, which facilitates the positioning in front of the image intensifier without affecting the measuring field for the $m A$ feedback loop. If the image intensifier can manually control the $\mathrm{mA}$ and $\mathrm{kV}$, then it can be used for measurement down to about $0.7 \mu \mathrm{Gy} / \mathrm{s}$. As a secondary parameter the total dose is accumulated, and after switching off the fluoroscopy, this value is used to calculate the average dose rate as the total dose divided by the exposure time. Figure 3 shows the physical specifications of the detector.

The inaccuracy is here defined as the root of the square sum of systematic errors, which has not been eliminated, and random errors (dispersion around a mean value). The calculation of the inaccuracy is based on 15 different measurements with a confidence level of $95 \%$. Of the total inaccuracy, random error is $20 \%$ and general inaccuracy is $80 \%$. As a reference condition to the IEC61674 standard and inside the Piranha top panel, the calibration is done with a field size typically $5 \mathrm{~mm}$ less than the size of the top panel.

The standard calibration for the Piranha External Dose Probe is W/23 mm Al. This calibration was chosen because the main use of the detector is to measure the dose by the image intensifier after the phantom study. However, it can just be used for measurements of skin dose as well. The detector reacts very linearly to energy response and is not affected by different filtrations. 
Fig. 2 a The C-arm apparatus of fluoroscopy used. $\mathbf{b}$ The lead plate at the thickness of $0.2 \mathrm{~mm}$ by $10 \mathrm{~cm} \times 10 \mathrm{~cm}$ size. $c$ The settings of alpha phantom in experiment. d The phantom, piranha, and filter array in experiment $\mathbf{a}$

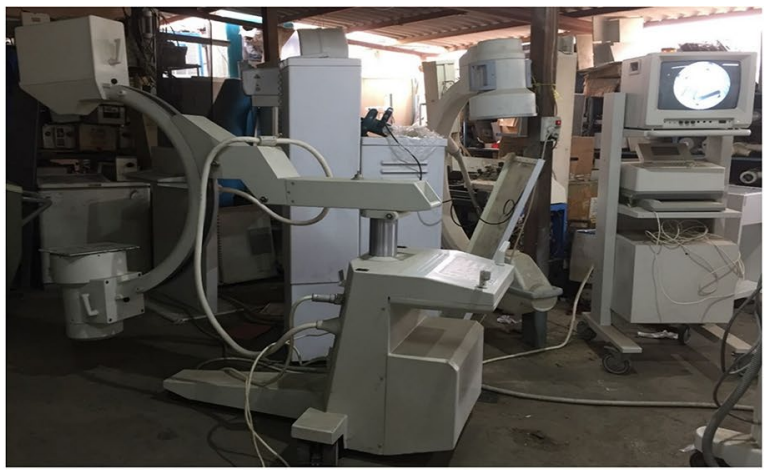

b

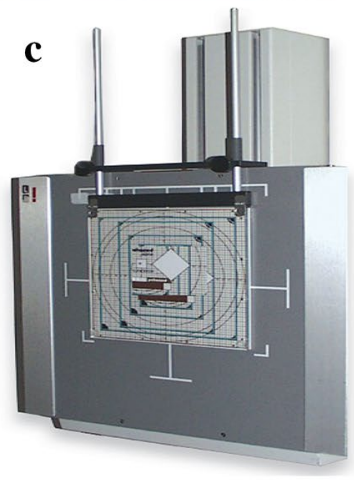

d

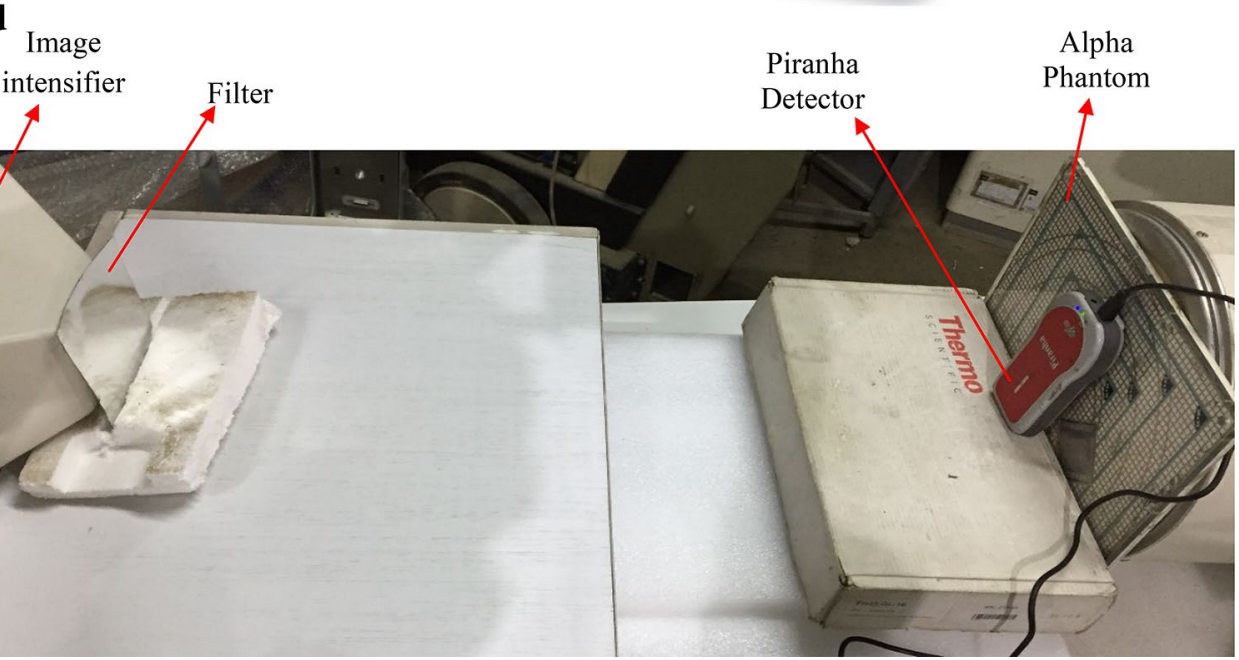

\begin{tabular}{|l|l|}
\hline Detector area & $3 \times 21.1 \mathrm{~mm}$ \\
\hline Detector position & $\begin{array}{l}10 \mathrm{~mm} \text { below top panel, as indicated in figure below and by a } 3 \mathrm{~mm} \text { rim } \\
\text { on } 3 \text { edges. }\end{array}$ \\
\hline Size & $133 \times 75 \times 26 \mathrm{~mm}\left(5.2^{\prime \prime} \times 2.9^{\prime \prime} \times 1.02 "\right)$ \\
\hline Weight & Approximately $405 \mathrm{~g}$ \\
\hline
\end{tabular}

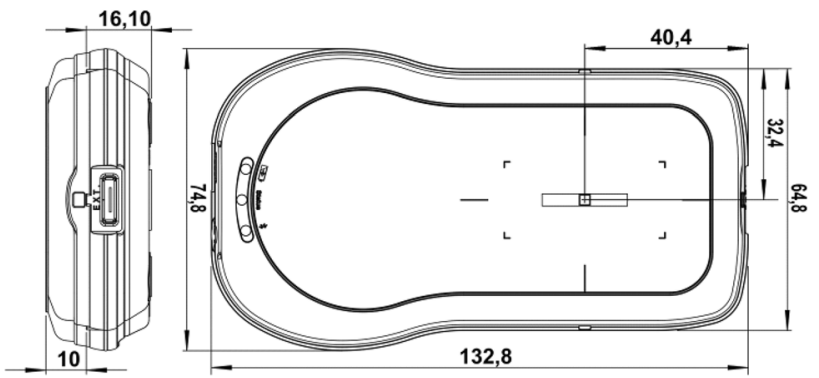

Fig. 3 Physical dimensions of Piranha detector
The dose rate was $4 \mathrm{nGy} / \mathrm{s}$ to $150 \mathrm{mGy} / \mathrm{s}$ with $\pm 5 \%$ or $\pm 1 \mathrm{nGy} / \mathrm{s}$ inaccuracy, and a resolution of $\pm 200 \mathrm{pGy} / \mathrm{s}$ by free-run or timed. Also, the irradiation time range was $0.1 \mathrm{~ms}$ to $34,000 \mathrm{~s}$ with $\pm 1 \%$ or $\pm 0.5 \mathrm{~ms}$ inaccuracy and $0.5 \mathrm{~ms}$ resolution.

\subsection{Phantom study}

The alpha phantom has been designed to test relative positions of the X-ray field, light beam diaphragm and vertical $X$-ray beam alignment, all in one exposure. According to Fig. 2c, it has 3 main sections: 1 . Main section of the Alpha phantom for testing synchronicity of the radiation field to that of the Light Beam Diaphragm. 2. Center Tube fixes to the Alpha phantom to check the accuracy of the

\section{SN Applied Sciences}


Vertical Beam Alignment. 3. Bucky Wall Stand Holder is an adjustable hanger that supports the alpha phantom on the face of a vertical Bucky.

By using the phantom, since the external dose probe (Piranha Dose Probe) is not sensitive to back scatter, a lower value compared to a transmission ion chamber is typically detected (typically in the range of 5-20\%). Moreover, the beam correction factor can be used to make automatic corrections. This correction factor may also be stored permanently in a Favorite for easy access.

Here according to Fig. $2 d$, the radiation detector was placed in the middle of the imaged area and in the middle of the phantom field to obtain the phantom image and to investigate the filter impact. The image quality was appraised with a fairly standard phantom in high and low contrast resolution to compute the patient skin entrance dose.

\section{Outcomes}

Figure 4 shows the achieved X-ray spectra with and without utilizing $0.1 \mathrm{~mm}$ lead filter via attenuation formula and initial flux. For energies greater than $90 \mathrm{keV}$ and to increase the attenuation, a thicker filter like $0.2 \mathrm{~mm}$ may be used, but this leads to a significant decrease in the intensity of the useful radiation. In order to approximate the thickness effect, the thickness of the lead was assumed to be $0.2 \mathrm{~mm}$. Therefore, by re-computing $e^{-\mu x}$ for different energies, the output intensities in the range of $45-120 \mathrm{keV}$ can be resolved. If one compares the intensities for the case with the $0.2 \mathrm{~mm}$ thick lead filter and the case with $0.1 \mathrm{~mm}$, it is resolved with increasing thickness that the damping ratio for high energies increases. For instance, the output

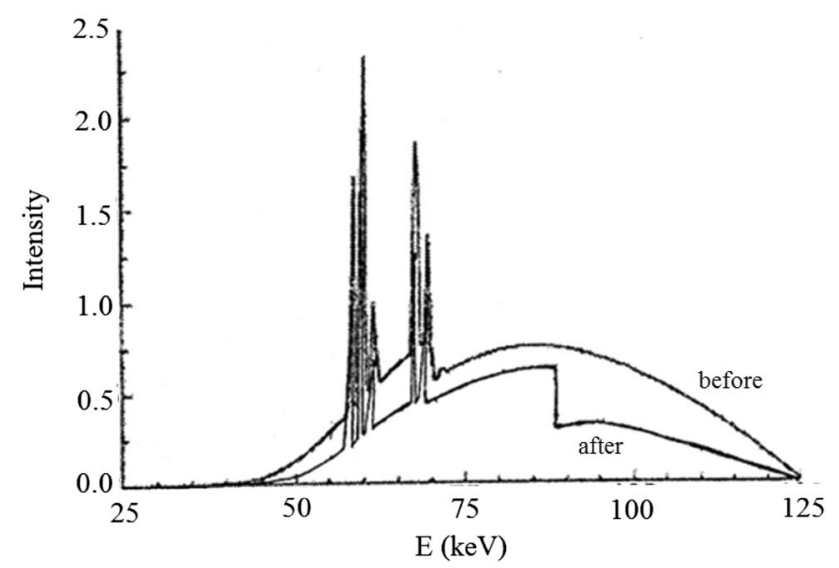

Fig. 4 Comparison of two X-ray spectra before (top) and after (bottom) filter assignment intensity ratio (OIR) of $0.2 \mathrm{~mm}$ filter to $0.1 \mathrm{~mm}$ filter will be 0.75 and 0.48 in $80 \mathrm{keV}$ and $95 \mathrm{keV}$, respectively from:

$\mathrm{OIR}_{\text {lead }}=\left(\frac{I_{0.2}}{I_{0.1}}\right)_{E}$

This means that as the thickness of the filter increases, the attenuation increases more at high energies than at low energies. It should be noted that increasing the thickness would decrease the intensity of the useful energy. We also found that, in accordance with the calculations, strong attenuation occurs at high and low energies immediately after the lead absorption peak by utilizing the filter at high energies.

By the MCNP4C code, the general geometry of the X-ray tube, the position of the filters in different thickness, and the photons and electrons generated by the characteristic and bremsstrahlung radiations were simulated for 10 million particles. The total simulation error was less than $1.5 \%$, which is an indication of accurate results. The outcomes given in Table 1 demonstrate the particles counted by 0.1 to $0.7 \mathrm{~mm}$ thick per energy after assignment of the lead filter.

Figure 5 shows the output X-ray spectra after assignment of the lead filter in diverse thicknesses. The outcomes showed that the desired results are achieved by setting the lead filter to a thickness of $0.1 \mathrm{~mm}$ and dismissing high and low energy photons. In the meantime, the useful part of the spectrum was retained and agreed with the lead absorption edge curve at $75 \mathrm{keV}$. Subsequently, a strong decrease of the particle numbers was followed. Meanwhile, increasing the filter thickness leads to more attenuation, that is undesirable to produce an image. Due to the consideration of the pure tungsten target and the lack of accurate information about the filters of the intrinsic tubes, there was no match between the X-ray output curves and the computed curves. Lastly, after executing the simulations under these circumstances, the desired results were obtained at a thickness of $0.1 \mathrm{~mm}$, which was consistent with the computation outputs. The contrast and quality of the image were low due to the utilizing high voltage, and the absorbed dose was also on a large scale. Although the resolution of the image remained unchanged by using of the lead filter, the quality of the image and contrast improved significantly, and the absorbed dose decreased considerably, so that the lead filter with thickness of $0.2 \mathrm{~mm}$ lost its effects. Therefore, the thickness of the lead filter was raised to $0.4 \mathrm{~mm}$ to enhance the quality and lessen the absorbed dose. The use of $0.6 \mathrm{~mm}$ of the lead filter reduced the resolution, contrast, and image quality. In the other phase, $0.2 \mathrm{~mm}$ tinplate and $0.2 \mathrm{~mm}$ lead plate were employed, which did not influence the quality and contrast but significantly lessened the patient's absorbed 
Table 1 Number of simulated output photons per starting particle at diverse thickness of the lead filter

\begin{tabular}{|c|c|c|c|c|c|c|c|}
\hline \multirow[t]{2}{*}{$\mathrm{E}(\mathrm{keV})$} & \multicolumn{7}{|c|}{ Thickness (mm) } \\
\hline & $0.1 \mathrm{~mm}$ & $0.2 \mathrm{~mm}$ & $0.3 \mathrm{~mm}$ & $0.4 \mathrm{~mm}$ & $0.5 \mathrm{~mm}$ & $0.6 \mathrm{~mm}$ & $0.7 \mathrm{~mm}$ \\
\hline 5.00 & 0 & 0 & 0 & 0 & 0 & 0 & 0 \\
\hline 8.06 & 0 & 0 & 0 & 0 & 0 & 0 & 0 \\
\hline 11.13 & 0 & 0 & 0 & 0 & 0 & 0 & 0 \\
\hline 14.19 & 0.09 & 0.02 & 0.02 & 0 & 0 & 0 & 0 \\
\hline 17.26 & 0 & 0 & 0 & 0.02 & 0 & 0 & 0 \\
\hline 20.32 & 0 & 0 & 0.04 & 0 & 0 & 0 & 0 \\
\hline 23.39 & 0.02 & 0 & 0 & 0 & 0 & 0 & 0 \\
\hline 26.45 & 0.03 & 0 & 0 & 0 & 0 & 0 & 0 \\
\hline 29.52 & 0 & 0 & 0 & 0 & 0 & 0 & 0 \\
\hline 32.58 & 0.02 & 0 & 0 & 0 & 0 & 0 & 0 \\
\hline 35.64 & 0.11 & 0 & 0 & 0 & 0 & 0 & 0 \\
\hline 38.71 & 0.16 & 0.02 & 0.02 & 0 & 0 & 0 & 0 \\
\hline 41.77 & 0.38 & 0.05 & 0.02 & 0 & 0 & 0 & 0 \\
\hline 44.84 & 0.18 & 0.04 & 0.04 & 0 & 0 & 0 & 0 \\
\hline 47.90 & 0.20 & 0.03 & 0.02 & 0 & 0 & 0 & 0 \\
\hline 50.97 & 0.41 & 0.13 & 0.05 & 0.02 & 0 & 0 & 0 \\
\hline 54.03 & 0.41 & 0.20 & 0.03 & 0 & 0 & 0 & 0 \\
\hline 57.10 & 0.56 & 0.23 & 0.10 & 0.05 & 0.05 & 0 & 0 \\
\hline 60.16 & 0.65 & 0.35 & 0.22 & 0.13 & 0.05 & 0 & 0 \\
\hline 63.23 & 0.46 & 0.16 & 0.10 & 0.03 & 0.02 & 0 & 0 \\
\hline 66.29 & 0.20 & 0.20 & 0.13 & 0.10 & 0.07 & 0.02 & 0.02 \\
\hline 69.36 & 0.53 & 0.25 & 0.20 & 0.15 & 0.05 & 0 & 0 \\
\hline 72.42 & 0.41 & 0.30 & 0.21 & 0.11 & 0.05 & 0 & 0 \\
\hline 75.49 & 0.41 & 0.30 & 0.41 & 0.23 & 0.09 & 0.02 & 0.02 \\
\hline 78.55 & 0.66 & 0.58 & 0.09 & 0.07 & 0.03 & 0.03 & 0.02 \\
\hline 81.61 & 0.33 & 0.24 & 0.20 & 0.12 & 0.05 & 0.05 & 0.02 \\
\hline 84.68 & 0.14 & 0.13 & 0.10 & 0.09 & 0.05 & 0.03 & 0 \\
\hline 87.74 & 0.13 & 0.13 & 0.22 & 0.17 & 0.03 & 0.05 & 0.05 \\
\hline 90.81 & 0.20 & 0.19 & 0.08 & 0.09 & 0.02 & 0 & 0 \\
\hline 93.87 & 0.06 & 0.03 & 0.02 & 0 & 0 & 0 & 0 \\
\hline 96.94 & 0.03 & 0.03 & 0.02 & 0 & 0 & 0 & 0 \\
\hline 100.00 & 0.06 & 0.02 & 0.02 & 0 & 0 & 0 & 0 \\
\hline
\end{tabular}

Fig. 5 The obtained X-ray spectra utilizing lead plates at diverse thicknesses

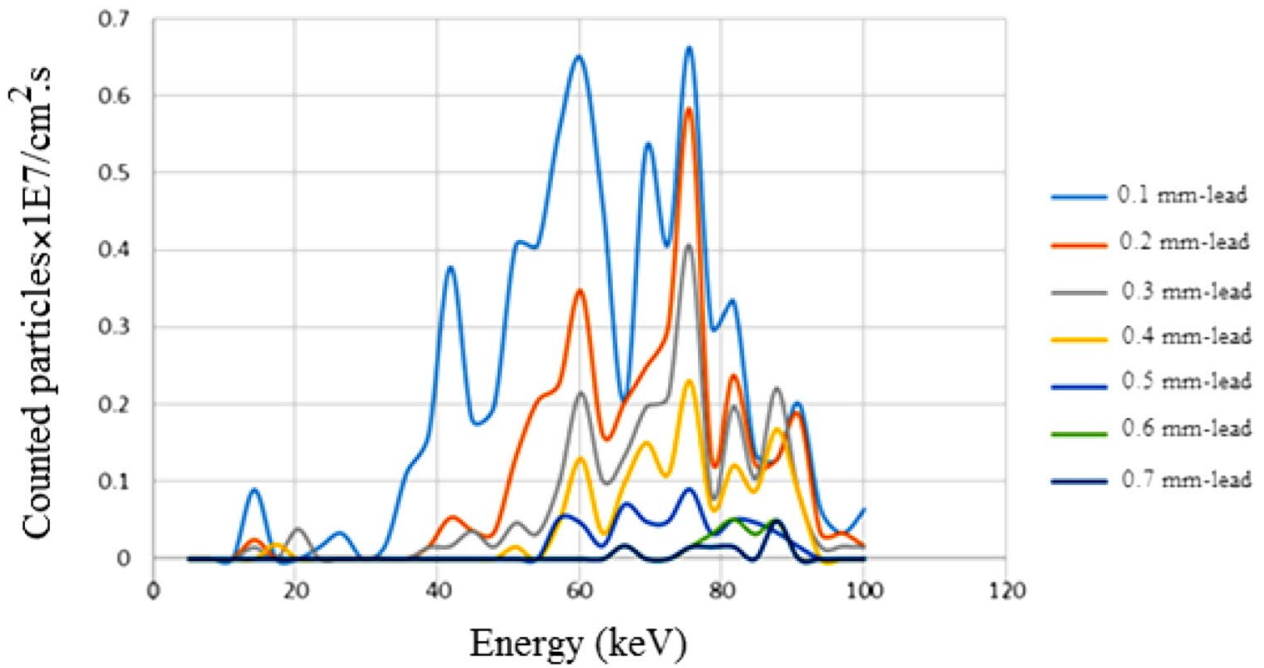


dose. By calculations, the selection of a $0.2 \mathrm{~mm}$ lead filter in this energy range was mathematically the right choice. So in order to get a high quality image, besides raising the $\mathrm{mAs}$, the lead filter should be thicker. Figure 6 illustrates the acquired images of an alpha phantom with and without lead filter having a thickness of $0.2 \mathrm{~mm}$ at a voltage of $100 \mathrm{keV}$ and $0.2,1.2$ and $2.7 \mathrm{~mA}$. If the filter was not used, the image became whiter by increasing the current. For this energy range used, the HVL for the lead plates with a thickness of $0.2 \mathrm{~mm}$ and $0.4 \mathrm{~mm}$ corresponded to that of aluminum with a thickness of $10.1 \mathrm{~mm}$ and $11.3 \mathrm{~mm}$, respectively. Table 2 demonstrates the dose ratio and the image resolution before and after the assignment of the tin filter. Without a filter, raising the $\mathrm{mAs}$ had not influence on the resolution, but on the image quality. Nevertheless the resolution preserved constant and the image quality enhanced well, the absorbed dose was significantly decreased. In addition, Fig. 7 shows the absorbed dose ratio in terms of intensity at $100 \mathrm{keV}$ energy. While the tin filter was used, the dose ratio acquired was markedly diminished by 50 and $250 \mu \mathrm{Gy} / \mathrm{s}$ for 0.5 and $3 \mathrm{~mA}$, correspondingly.

Haga et al. [31] have used three copper attenuators by different thickness of $0.5,1.5$ and $3 \mathrm{~mm}$ in a rotatable panel to evaluate the image performance in a quality control test. They discovered the spatial resolution in the thin attenuation setting $(0.5 \mathrm{~mm} \mathrm{Cu})$ was higher than that in the $3.0 \mathrm{~mm} \mathrm{Cu}$. In interventional radiology with several fluoroscopes, the stored fluoro loops are only saved after multiple activations of the fluoro pedal to achieve steady state dosimetric parameters ( $k \mathrm{p}, \mathrm{mA}$, spectral filter). Therefore, a method often is described as a "double clutch"
Fig. 6 a The obtained alpha phantom image without using filter by $100 \mathrm{keV}$ and $0.2 \mathrm{~mA}$ (Left). The obtained image using $0.2 \mathrm{~mm}$ lead plate (Right). b The obtained alpha phantom image without using filter by $100 \mathrm{keV}$ and $1.2 \mathrm{~mA}$ (Left). The obtained image using $0.2 \mathrm{~mm}$ lead plate (Right). c The obtained alpha phantom image without using filter by $100 \mathrm{keV}$ and $2.7 \mathrm{~mA}$ (Left). The obtained image using $0.2 \mathrm{~mm}$ lead plate (Right) a

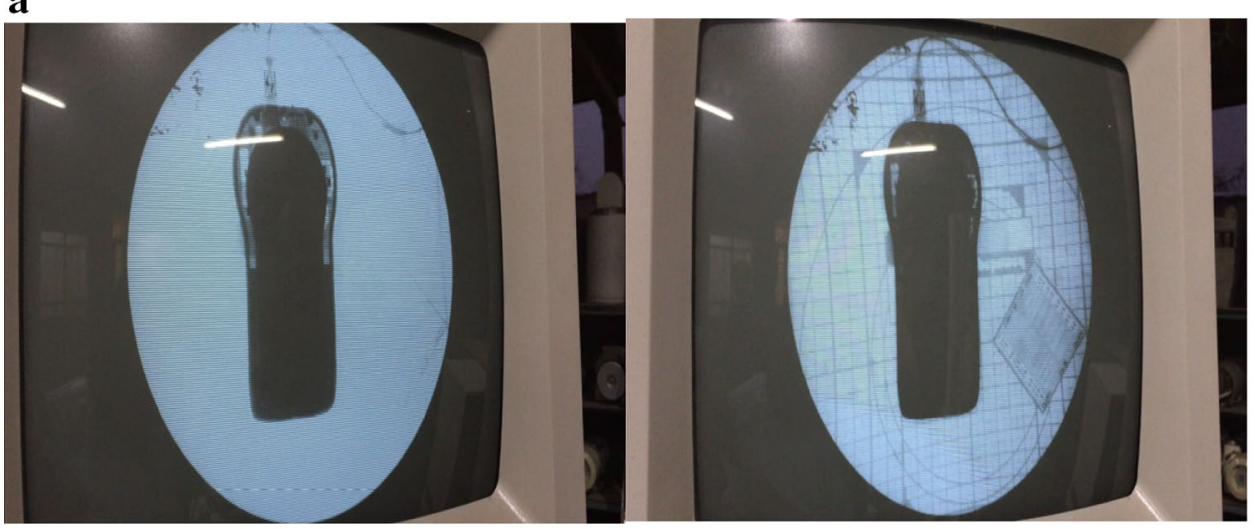

b

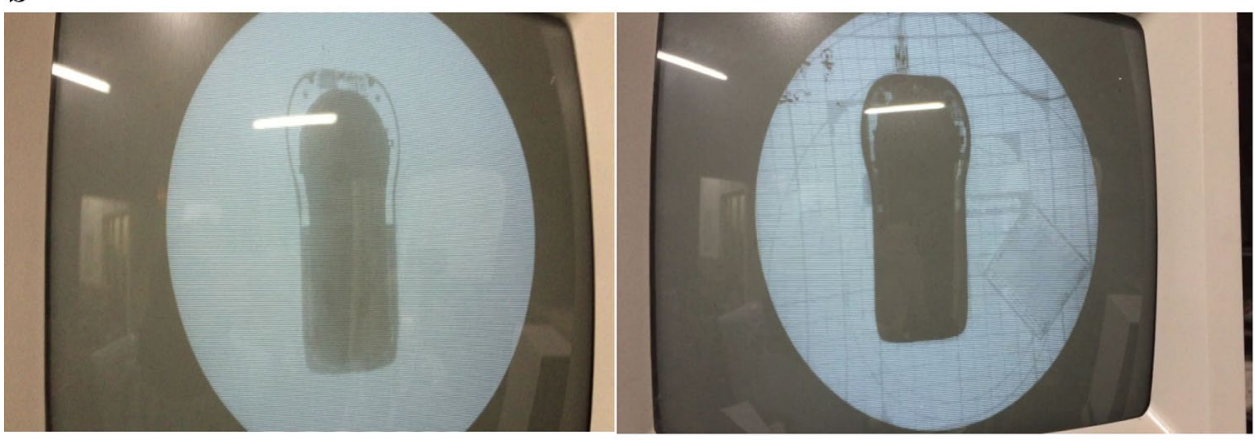

c

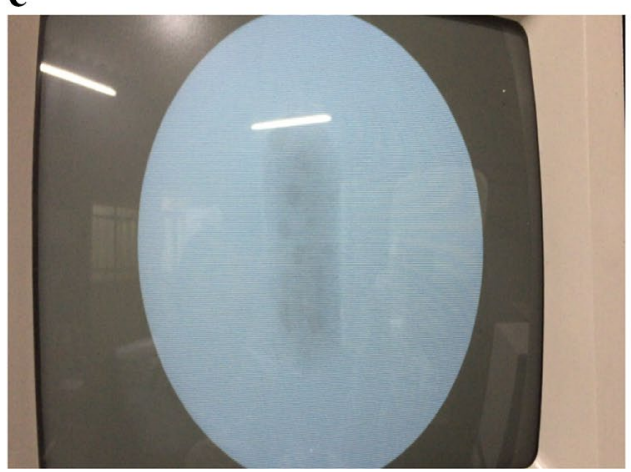

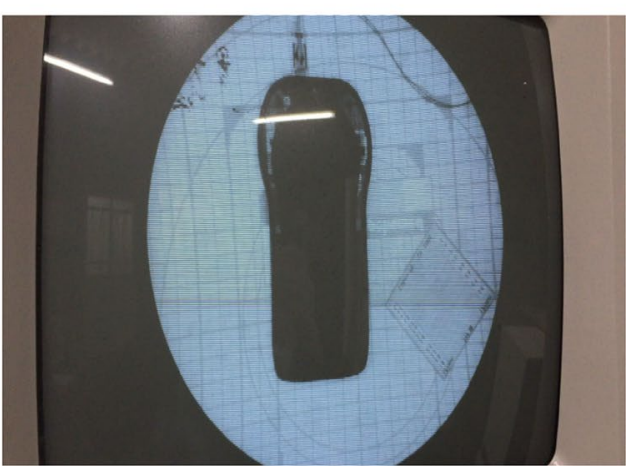

SN Applied Sciences A SPRINGER NATURE journa 
Table 2 Outcomes experimented using and without using tin filter

\begin{tabular}{|c|c|c|c|c|c|}
\hline \multicolumn{3}{|c|}{ With the use of tin filter } & \multicolumn{3}{|c|}{ Without the use of tin filter } \\
\hline $\begin{array}{l}\text { Intensity } \\
\text { (mAs) }\end{array}$ & Resolution & Dose ratio $(\mu \mathrm{Gy} / \mathrm{s})$ & $\begin{array}{l}\text { Thickness } \\
(\mathrm{mm})\end{array}$ & Resolution & $\begin{array}{l}\text { Dose ratio } \\
(\mu \mathrm{G} / \mathrm{s})\end{array}$ \\
\hline 0.1 & 1 & 29.19 & 0.2 & 1 & 2.30 \\
\hline 0.2 & 1 & 34.21 & 0.2 & 1 & 2.54 \\
\hline 0.3 & 1 & 36.52 & 0.2 & 1 & 2.70 \\
\hline 0.4 & 1 & 44.93 & 0.2 & 1 & 2.99 \\
\hline 0.6 & 1 & 45.01 & 0.2 & 1 & 3.26 \\
\hline 0.8 & 1 & 52.58 & 0.2 & 1 & 3.90 \\
\hline 1.0 & 1 & 83.85 & 0.2 & 1 & 5.97 \\
\hline 1.2 & 1 & 107.81 & 0.2 & 1 & 7.32 \\
\hline 1.4 & 1 & 120.90 & 0.2 & 1 & 7.86 \\
\hline 1.6 & 1 & 126.55 & 0.2 & 1 & 9.53 \\
\hline 1.8 & 1 & 142.14 & 0.4 & 1 & 2.90 \\
\hline 2.0 & 1 & 168.93 & 0.4 & 1 & 3.20 \\
\hline 2.2 & 1 & 212.71 & 0.4 & 1 & 3.30 \\
\hline 2.4 & 1 & 220.83 & 0.4 & 1 & 3.64 \\
\hline 2.7 & 1 & 249.71 & 0.4 & 1 & 4.22 \\
\hline 3.0 & 1 & 250.52 & 0.4 & 1 & 4.87 \\
\hline
\end{tabular}

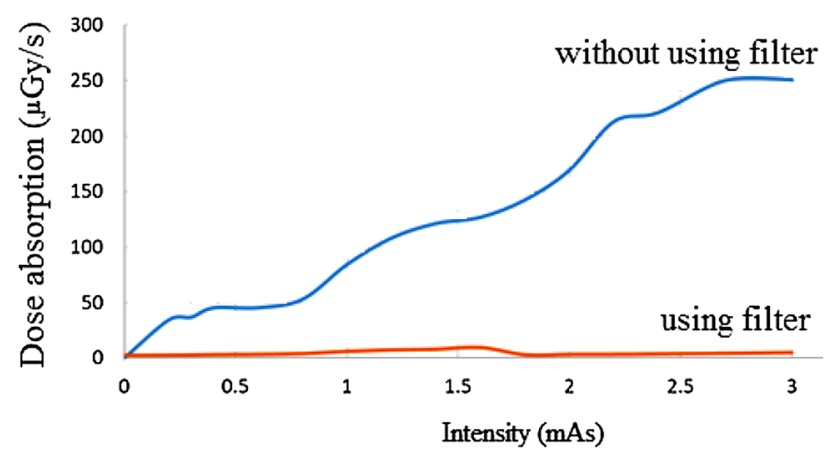

Fig. 7 The acquired absorbed dose ratio in terms of intensity by $100 \mathrm{keV}$ without and with the use of tin plate

so that a possible spectral filter or other parameters may change as needed due to increased or different phantom loading. Goode et al. [32] have been utilized $0.3 \mathrm{~mm} \mathrm{Cu}$ spectral filter in a normal fluoro dose level and to reach a low dose setting with high beam quality, the copper spectral filter increased $0.6 \mathrm{~mm}$ besides lessening $5 \mathrm{mG} / \mathrm{min}$ dose rate and growing $\mathrm{kVp}$ by 3 . They indicated that the signal to noise ratio (SNR) is increased from normal to low dose level for a thickened phantom. Furthermore, Ngaile et al. [33] have reported that $0.35 \mathrm{~mm}$ thickness of copper can be used in fluoroscopy without significant impact on the image quality, and using $0.35 \mathrm{~mm}$ Cu plus $1 \mathrm{~mm} \mathrm{Al}$ additional filtration preserves the contrast by $71 \%$ reduction in surface air Kerma.

The limitations of our research are that only one supplier of fluoroscopes and a small selection of models were available for these tests. Future work will include at least two more fluoroscopy suppliers and several other models to evaluate the filtration influence on image quality. Through a performance test with a simple patient-equivalent phantom, the quality control criteria can be further examined to design a distinct fluoroscope spectrum via diverse or complex filters.

\section{Conclusions}

In fact, extra filtration has the potential to change the $X$-ray spectrum, which often brings benefits in terms of patient dose and image quality. It is recommended to use the band pass filter to obtain high quality images, which should be determined by the attenuation profiles of the different materials. The work area in radiology, the X-ray spectrum peak, and the absorption edge are the most important factors in filter selection. For best outcomes, increasing the filter thickness also requires raising the $\mathrm{mAs}$ or exposure time. Because the spectrum peak is at $88 \mathrm{keV}$, utilizing the lead filter creates a narrow spectrum to enhance the image quality and decrease the absorbed patient dose. As the increase in thickness results in quality degradation, the $\mathrm{mAs}$ are raised as much as possible to obtain a high quality image. The results of experiments, simulations and calculations showed that lead submission at $100 \mathrm{kVp}$ can lessen the tube output dose and a boost in $\mathrm{mA}$ can enhance the image quality while the dose is still lower than that of the unfiltered instance. By virtue of the 
outcomes, the $0.1 \mathrm{~mm}$ thick lead filter was preferred as the optimal filter.

\section{Compliance with ethical standards}

Conflict of interest The authors declare that they have no conflict of interest.

\section{References}

1. Loffroy R, Lin M, Rao P, Bhagat N, Noordhoek N, Radaelli A, Blijd J, Geschwind JF (2012) Comparing the detectability of hepatocellular carcinoma by C-arm dual-phase cone-beam computed tomography during hepatic arteriography with conventional contrast-enhanced magnetic resonance imaging. Cardiovasc Intervent Radiol 35:97-104. https://doi.org/10.1007/s0027 0-011-0118-x

2. Taghizadeh Delkhoush C, Maroufi N, Ebrahimi Takamjani I, Farahmand F, Shakourirad A, Haghani H (2014) Dynamic comparison of segmentary scapulohumeral rhythm between athletes with and without impingement syndrome. Iran J Radiol 11(2):e14821. https://doi.org/10.5812/iranjradiol.14821

3. Khatami A, Araghi S, Babaei T (2019) Evaluating the performance of different classification methods on medical X-ray images. SN Appl Sci 1:1154. https://doi.org/10.1007/s42452-019-1174-0

4. Boroujeni FZ, Rahmat RWOK, Mustapha N, Affendey LS, Maskon O (2011) Automatic selection of initial points for exploratory vessel tracing in fluoroscopic images. Def Sci J 61(5):443-451

5. Asgari A, Ashoor M, Sarkhosh L, Khorshidi A, Shokrani P (2019) Determination of Gamma Camera's calibration factors for quantitation of diagnostic radionuclides in simultaneous scattering and attenuation correction. Curr Radiopharm 12(1):29-39. https ://doi.org/10.2174/1874471011666180914095222

6. Savareh BA, Sadat Y, Bashiri A, Shahi M, Davaridolatabadi N (2017) The design and implementation of the software tracking cervical and lumbar vertebrae in spinal fluoroscopy images. Future Sci OA 3(4):FSO240. https://doi.org/10.4155/ fsoa-2017-0089

7. Khorshidi A (2018) Accelerator-based methods in radio-material 99Mo/99mTc production alternatives by monte carlo method: the scientific-expedient considerations in nuclear medicine. J Multiscale Model 10:1930001. https://doi.org/10.1142/S1756 973719300016

8. Toufan M, Pourafkari L, Nader ND (2017) Right ventricular perforation as a complication of fluoroscopy-guided pericardiocentesis. Rev Port Cardiol 36(6):479-480. https://doi.org/10.1016/j. repc.2016.05.013

9. Jalali M, Farahmand F, Mousavi SM, Golestanha SA, Rezaeian T, Shirvani Broujeni S, Rahgozar M, Esfandiarpour F (2015) Fluoroscopic analysis of tibial translation in anterior cruciate ligament injured knees with and without bracing during forward lunge. Iran J Radiol 12(3):e17832. https://doi.org/10.5812/iranjradio I.17832v2

10. Hashemi M, Mofrad MK, Mohajerani SA, Kazemi SM, Radpey B, Zali A (2015) Anatomical flow pattern of contrast in lumbar epidural space: a human study with a midline vs. parasagittal interlaminar approach under fluoroscopy. Pain Physician 18(4):317-324

11. Motiei-Langroudi R, Sadeghian H (2015) Assessment of pedicle screw placement accuracy in thoracolumbosacral spine using freehand technique aided by lateral fluoroscopy: results of postoperative computed tomography in 114 patients. Spine J 15(4):700-704. https://doi.org/10.1016/j.spinee.2014.12.012

12. Nabipour JS, Khorshidi A (2018) Spectroscopy and optimizing semiconductor detector data under $X$ and $Y$ photons using image processing technique. J Med Imaging Radiat Sci 49(2):194-200. https://doi.org/10.1016/j.jmir.2018.01.004

13. Khorshidi A, Ashoor M, Hosseini SH, Rajaee A (2012) Evaluation of collimators' response: round and hexagonal holes in parallel and fan beam. Prog Biophys Mol Biol 109(3):59-66. https://doi. org/10.1016/j.pbiomolbio.2012.03.003

14. Khorshidi A, Ashoor M (2014) Modulation transfer function assessment in parallel beam and fan beam collimators with square and cylindrical holes. Ann Nucl Med 28(4):363-370. https ://doi.org/10.1007/s12149-014-0820-2

15. Khorshidi A, Ashoor M, Hosseini SH, Rajaee A (2012) Estimation of fan beam and parallel beam parameters in a wire mesh design. J Nucl Med Technol 40(1):37-43. https://doi. org/10.2967/jnmt.111.089904

16. Khorshidi A (2019) Radiochemical parameters of molybdenum-99 transmutation in cyclotron-based production method using a neutron activator design for nuclear-medicine aims. Eur Phys J Plus 134:249. https://doi.org/10.1140/epjp/i2019-12568 $-3$

17. Leschka $S$, Stolzmann $P$, Baumüller $S$, Scheffel $H$, Desbiolles L, Schmid B, Marincek B, Alkadhi H, Metrics P (2010) Performance of dual-energy $C T$ with tin filter technology for the discrimination of renal cysts and enhancing masses. Acad Radiol 17(4):526-534. https://doi.org/10.1016/j.acra.2009.11.007

18. Suntharalingam $S$, Allmendinger T, Blex $S$, Al-Bayati M, Nassenstein K, Schweiger B, Forsting M, Wetter A (2017) Spectral beam shaping in unenhanced chest $C T$ examinations: a phantom study on dose reduction and image quality. Acad Radiol 25(2):153-158. https://doi.org/10.1016/j.acra.2017.08.011

19. May MS, Brand M, Lell MM, Sedlmair M, Allmendinger T, Uder $M$, Wuest W (2017) Radiation dose reduction in parasinus CT by spectral shaping. Neuroradiology 59(2):169-176. https://doi. org/10.1007/s00234-016-1780-0

20. Kim SC, Dong KR, Chung WK (2012) Medical radiation shielding effect by composition of barium compounds. Ann Nucl Energy 47:1-5. https://doi.org/10.1016/j.anucene.2012.04.014

21. Nicholson R, Tuffee F, Uthappa CM (2000) Skin sparing in interventional radiology: the effect of copper filtration. $\mathrm{Br} J$ Radiol 73:36-42. https://doi.org/10.1259/bjr.73.865.10721318

22. Moey SF, Shazli ZA, Sayed I (2017) Dose evaluation for common digital radiographic examinations in selected hospitals in Pahang Malaysia. Iran J Med Phys 14:155-161. https://doi. org/10.22038/ijmp.2017.22744.1220

23. Ashoor M, Khorshidi A (2019) Evaluation of crystals' morphology on detection efficiency using modern classification criterion and monte carlo method in nuclear medicine. Proc Natl Acad Sci USA India Sect A Phys Sci 89(3):579-585. https://doi. org/10.1007/s40010-018-0482-x

24. Matthews K, Brennan PC (2009) Optimisation of X-ray examinations: general principles and an Irish perspective. Radiography 15(3):262-268. https://doi.org/10.1016/j.radi.2008.07.002

25. Prince $E$ (2004) International tables for crystallography, volume C: mathematical, physical and chemical tables. Springer, Berlin

26. Allison JW (1961) Gamma-radiation absorption coefficients of various materials allowing for bremsstrahlung and other secondary radiations. Aust J Phys 14:443-468

27. Khorshidi A, Pazirandeh A (2018) Molybdenum transmutation via $98 \mathrm{Mo}$ samples using bismuth/lead neutron moderators. Eurphys Lett 123(1):12001. https://doi. org/10.1209/0295-5075/123/12001

28. Khorshidi A (2018) Neutron activator design for 99Mo production yield estimation via lead and water moderators in 
transmutation's analysis. Instrum Exp Tech 61(2):198-204. https ://doi.org/10.1134/S002044121802015X

29. Das $S$ (2019) Recent advances in characterising irradiation damage in tungsten for fusion power. SN Appl Sci 1:1614. https://doi. org/10.1007/s42452-019-1591-0

30. Khorshidi A (2016) Gold nanoparticles production using reactor and cyclotron based methods in assessment of 196,198Au production yields by 197Au neutron absorption for therapeutic purposes. Mater Sci Eng C 68(1):449-454. https://doi. org/10.1016/j.msec.2016.06.018

31. Haga Y, Chida K, Inaba Y, Kaga Y, Meguro T, Zuguchi Z (2016) A rotatable quality control phantom for evaluating the performance of flat panel detectors in imaging moving objects. J Digit Imaging 29(1):38-42. https://doi.org/10.1007/s1027 8-015-9816-2

32. Goode AR, Snyder C, Snyder A, Collins P, DeLorenzo M, Lin PJ (2019) Signal and contrast to noise ratio evaluation of fluoroscopic loops for interventional fluoroscope quality control. J
Appl Clin Med Phys 20(10):172-180. https://doi.org/10.1002/ acm2.12734

33. Ngaile JE, Msaki PK, Kazema RR, Mwimanzi JM, Mango M (2019) Evaluation of the influence of additional beam filtration on image quality and patient dose in $\mathrm{x}$-ray fluoroscopy procedures. Tanzan J Sci 45(2):253-264

Publisher's Note Springer Nature remains neutral with regard to jurisdictional claims in published maps and institutional affiliations. 